Thierry Boulain

Maurizio Cecconi

\section{Can one size fit all? The fine line between fluid overload and hypovolemia}

Received: 15 January 2015

Accepted: 26 January 2015

Published online: 6 February 2015

(C) Springer-Verlag Berlin Heidelberg and ESICM 2015

\section{T. Boulain (凶)}

Medical-Surgical Intensive Care Unit, Hôpital de La Source, Centre Hospitalier Régional d'Orléans, BP 6709,

45067 Orléans cedex, France

e-mail: thierry.boulain@chr-orleans.fr

Tel.: 33238514446

\section{Cecconi}

Anaesthesia and Intensive Care, St George's Hospital and Medical School, London, UK

In septic shock like in other types of shock, hypovolemia, whether caused by a true loss of circulating blood volume and/or by vasodilation, results in a decrease of the mean systemic filling pressure, the driving force for venous return to the heart, thereby leading to insufficient cardiac preload and output [1]. Rapid volume expansion using repeated intravenous fluid boluses is thus the mainstay intervention during the first hours of care [2]. Meanwhile, recent data in intensive care unit (ICU) patients [3], or in surgical patients [4], suggest that fluid and salt overload have negative impact on morbidity and mortality. Additionally, in patients with acute respiratory distress syndrome (ARDS), a restrictive fluid administration policy driven by an algorithm based on cardiac filling pressures has been shown to shorten the length of mechanical ventilation and of ICU stay. Therefore, it is essential to strike the right balance in order to not neglecting hypovolemia and its devastating consequences and in the meanwhile not worsening interstitial and pulmonary oedema (Fig. 1). The way fluid management is delivered is not standardized, as reflected by the high variability in the way fluid boluses are decided and guided in real life practice [5]. There is a lack of randomised controlled trials testing different strategies of volume expansion based on objectives measurements during the first days of septic shock. Neither the systematic use of the well-known (but rarely used in ICU practice [5]) fluid challenge technique [6-8] nor the systematic use of the few available predictive indices of fluid responsiveness $[9,10]$, have been tested against usual care with hard endpoints such as mortality. Conducting such trials is very challenging for physiological reasons (haemodynamic instability does not have a single cause and "one size fits all" algorithm are intrinsically deemed to fail) and for logistical reasons $(24 / 24,7 / 7$ availability of investigators; rigorous protocol and risk of protocol violation/deviation; need of large sample sizes).

In a study recently published in Intensive Care Medicine, Zhang et al. [11] courageously took on the challenge of comparing two strategies of fluid management in the

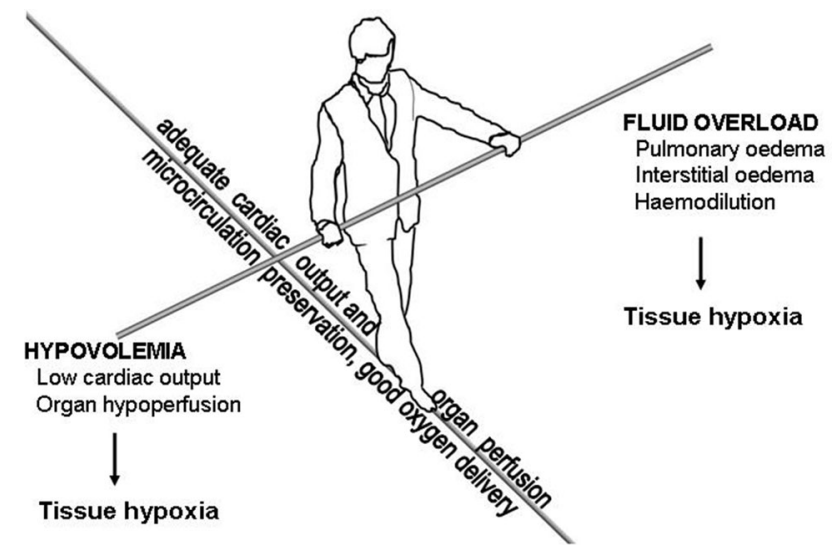

Fig. 1 The art of fluid management: Walking the fine line between the dangers of fluid overload and hypovolemia 
first days of care in patients with septic shock and/or ARDS and should be commended for this. They included 350 patients (75 with ARDS, 166 with septic shock, and 108 with both diseases) in a 1:1 randomised trial that compared day-28 all-cause mortality between patients treated according to an algorithm based on parameters derived from PICCO (Pulsion Medical Systems, Germany) monitoring [cardiac index, intrathoracic blood volume (ITBV) and extravascular lung water (EVLW)] and patients treated according to a central venous pressure (CVP)-based algorithm. Despite a stratified randomization process patients in the PICCO group had significantly higher baseline SOFA and APACHE II scores. This is somehow unfortunate as clearly the baseline severity of disease has an impact on the mortality however the authors corrected for these factors in their analysis. The day28 mortality was similar in the PICCO group (49\%) and the CVP group (50\%), and the trial was stopped early for reasons of futility. These results did not change when SOFA and APACHE II scores were input into a multivariate regression model. A big limitation of this study is probably that the same algorithm was applied for shock and ARDS. Since the treatment algorithm in the PICCO group was mainly centred on ITBV (to be maintained within an arbitrary although commonly used range, using fluid loading or furosemide) and EVLW (to be maintained below $10 \mathrm{ml} / \mathrm{kg}$ by fluid removal, either with furosemide or haemofiltration), a too quick but tempting interpretation of the results would be to conclude that the haemodynamic variables used add nothing to current practice. However no monitoring technique can cure patients. It is the treatment strategy/algorithm that ultimately can affect patients' outcome [12]. In practice this is not the impact of the PICCO system on patients' outcome that has been tested, but rather an arbitrary treatment algorithm. From a physiological point of view, as previously said, a "one size fits all" approach was probably deemed to fail. For instance, in the PICCO group, patients could undergo fluid removal by furosemide administration or haemofiltration as soon as Day 1 based solely on high ITBV. Moreover, ARDS patients without shock could alternate fluid removal when EVLW was above $10 \mathrm{ml} / \mathrm{kg}$ and fluid administration when ITBV was below $850 \mathrm{ml} / \mathrm{m}^{2}$. Undoubtedly, whatever the study arm considered, due to the thresholds chosen to trigger furosemide administration, patients were exposed to cycles of hypovolemia and hypervolemia.

EVLW measurements by single transpulmonary thermodilution have proved their accuracy and undoubtedly reflect the amount of lung oedema [13] and high EVLW is closely linked to ICU mortality in the most severely ill patients [14]. Despite the fact that the results of the Mitchell's et al. [15], study using EVLW of the double indicator with COLD system have not been confirmed with a single indicator yet, studies testing strategies based on EVLW in clinical practice should definitely be pursued but we should carefully design treatment algorithms before.

Because of the uncertainty about the effectiveness and safety of fluid boluses in most situations of shock once passed the first hours of resuscitation, and because there are no currently reliable clinical data documenting the midterm safety of a fluid bolus that would be useless in terms of cardiac output augmentation, it is advocated to reserve fluid bolus to patients with a priori reasonable probability of positive response in cardiac output, and to avoid it in patients with low probability of positive response [2]. To do this, dynamic predictive indices of fluid responsiveness are probably the most appropriate tools whereas static indices of cardiac preload (such as CVP or ITBV) have repeatedly shown their poor performance. However it has never been investigated whether they can be used to guidea "deresuscitation" strategy when the clinicians' aims are to achieve a negative fluid balance despite maintaining adequate perfusion.

To conclude we believe that the biggest limitation of this study is the fact that the algorithm was the same both in ARDS and in shock states. While ARDS can present with shock and patients in shock can often develop ARDS the therapeutic strategy may be very different. An algorithm where "one size fits all" does not seem to fit with the physiological understanding of a very dynamic situation such as the unstable critical patient [16]. This seems to be suggested also by the subgroup analysis where the trends for benefit or harm with the protocol are clearly in opposite directions in the ARDS and shock group.

In summary the authors have to be praised for the ambition of conducting this study but the only conclusions we can draw is that the used algorithm is not beneficial. It is also reassuring to see that one single algorithm cannot be used to treat every type of cardiovascular instability in the intensive care as it may lead to harm or benefit depending on the type of patient treated. Ultimately it is only knowledge and interpretation of the physiology at the bedside that can improve outcome.

Conflicts of interest M.C. has received consultation fees from Masimo, Edwards Lifesciences and LiDCO. T.B. has no conflict of interest to declare. 


\section{References}

1. Perner A, De Backer D (2014) Understanding hypovolaemia. Intensive Care Med 40:613-615

2. Cecconi M, De Backer D, Antonelli M et al (2014) Consensus on circulatory shock and hemodynamic monitoring. Task force of the European society of intensive care medicine. Intensive Care Med 40:1795-1815

3. Kox M, Pickkers P (2013) Less is more in critically III patients: not too intensive. JAMA Intern Med 173:1369

4. Raghunathan K, McGee WT, Higgins T (2012) Importance of intravenous fluid dose and composition in surgical ICU patients. Curr Opin Crit Care $18: 350-357$

5. Boulain T, Boisrame-Helms J, Ehrmann $S$ et al (2014) Volume expansion in the first 4 days of shock: a prospective multicentre study in 19 French intensive care units. Intensive Care Med. doi:10.1007/s00134-014-3576-1

6. Vincent JL, Weil MH (2006) Fluid challenge revisited. Crit Care Med 34:1333-1337
7. Cecconi M, Parsons AK, Rhodes A (2011) What is a fluid challenge? Curr Opin Crit Care 17:290-295

8. Cecconi M, Aya HD, Geisen $\mathrm{M}$ et al (2013) Changes in the mean systemic filling pressure during a fluid challenge in postsurgical intensive care patients. Intensive Care Med 39:1299-1305

9. Cavallaro F, Sandroni C, Marano C et al (2010) Diagnostic accuracy of passive leg raising for prediction of fluid responsiveness in adults: systematic review and meta-analysis of clinical studies. Intensive Care Med 36:1475-1483

10. Pinsky MR (2014) Functional haemodynamic monitoring. Curr Opin Crit Care 20:288-293. doi:10.1097/MCC.0000000000000090

11. Zhang Z, Ni H, Qian Z (2015)

Effectiveness of treatment based on PiCCO parameters in critically ill patients with septic shock and/or acute respiratory distress syndrome: a randomized controlled trial. Intensive Care Med. doi:10.1007/ s00134-014-3638-4
12. Pinsky MR, Payen D (2005) Functional hemodynamic monitoring. Crit Care 9:566-572

13. Neumann P (1999) Extravascular lung water and intrathoracic blood volume: double versus single indicator dilution technique. Intensive Care Med 25:216-219

14. Jozwiak M, Silva S, Persichini R et al (2013) Extravascular lung water is an independent prognostic factor in patients with acute respiratory distress syndrome. Crit Care Med 41:472-480

15. Mitchell JP, Schuller D, Calandrino FS et al (1992) Improved outcome based on fluid management in critically ill patients requiring pulmonary artery catheterization. Am Rev Respir Dis 145:990-998

16. Goldstein S, Bagshaw S, Cecconi M, ADQI XII Investigators Group et al (2014) Pharmacological management of fluid overload. Br J Anaesth 113:756-763 